\title{
Justification de la méthode des échelles multiples pour une classe d'équations aux dérivées partielles (*).
}

\author{
E. Sanchez-Palencla (Paris, France)
}

\begin{abstract}
Summary. - We study the two-seales method for the approximate resolution of a type of partial differential equations arising in the theory of slightly viscoelastic vibrations. An estimates of the error is given.
\end{abstract}

\section{1. - Introduction.}

Les méthodes de centrage [1] et échelles multiples [2] s'appliquent à la recherche de solutions approchées de problèmes de vibration comportant un petit paramètre $\varepsilon$ de perturbation qui produit une modulation lentement variable des vibrations.

Le but de ces méthodes est d'obtenir des solutions approchées valables dans des intervalles de temps longs, de l'ordre $\varepsilon^{-1}$ en général. La justification de la méthode de centrage pour les équations différentielles ordinaires est bien établie (cf. par exemple, [1], [3]) ; d'autre part, l'équivalence formelle des méthodes d'échelles multiples et centrage ([3], ef. aussi [4]) permet en conséquence de justifier les échelles multiples. Signalons pour mémoire que la justification du centrage se fait en général pour des équations écrites sous la "forme standard" où le paramètre $\varepsilon$ apparait en facteur du second membre.

Le centrage et les échelles multiples s'appliquent aussi à des systèmes régis par des équations aux dérivées partielles (ef. par exemple, [5] et [2]), mais la justificacation n'est faite à l'heure actuelle que de façon beaucoup moins satisfaisante. En effet, le centrage peut se faire et se justifier pour des équations différentielles dans des espaces de Banach (toujours sous forme standard, [3], [6], [7]), mais la réduction des équations aux dérivées partielles à des équations différentielles de la forme standard n'est pas toujours facile car elle fait intervenir des opérateurs non bornés, [6], [7], [8]. En fait, dans les applications on rencontre des équations aux dérivées partielles dont le terme de perturbation fait intervenir des dérivées d'ordre supérieur à celui de l'équation non perturbée [9], [10]; l'équation non perturbée est hyperbolique, tandis que l'équation perturbée est parabolique. Il est clair que dans ce cas, la réduction à une équation différentielle dans un espace de Banach ne peut se faire, car l'équation perturbée, parabolique, ne possède des solutions que pour $t>0$, alors

(*) Entrata in Redazione il 20 febbraio 1977. 
qu'une équation différentielle dans un espace de Banach a, en général, solution pour $t$ positif ou négatif.

Le but de cet article est de donner une justification de la méthode des échelles multiples pour un type d'équations linéaires de type parabolique-hyperbolique qui n'est pas de forme standard. Nous obtenons une estimation de l'erreur uniforme dans des intervalles de $t$ de l'ordre $\varepsilon^{-1}$ et, dans certains cas particuliers, pour tout $t>0$. Cette étude est motivée par un problème de vibrations viscoélastiques dont les calculs formels ont été donnés dans [9]. En fait, nous traitons ici un type d'équations un peu plus général que celui de [9], dont les calculs sont ainsi justifiés, au moins pour la première approximation, seule que nous étudions ici. La méthode d'estimation de l'erreur rappelle un peu celle utilisée dans [11] pour des équations différentielles ordinaires et un type assez restreint d'équations aux dérivées partielles. Signalons aussi qu'un problème analogue au nôtre, mains arec une hypothèse de commutativité des opérations $A$ et $B$ (cf. notre équation $(3,4)$ ) est traitée dans [12], sect. VI-6.

Le plan de l'article est le suivant:

Dans la section 2 nous présentons le type d'équations aux dérivées partielles auquel appartient le problème perturbé et nous énonçons un théorème d'existence et d'unicité. La sect. 3 est consacrée à l'énoncé du problème de perturbation et à sa formulation dans la base formée par les fonctions propres de l'équation non perturbée. La mise en oeuvre formelle de la méthode des échelles multiples qu'il s'agit de justifier est faite dans la section 4, à la fin de laquelle on énonce aussi le résultat d'estimation d'erreur dans le cas où un seul mode propre est excité à l'instant initial. Les sect. 5 et 6 contiennent la démonstration de cette estimation (une description rapide du raisonnement employé se trouve au début de la section 5). Le cas général où tous les modes sont excités à l'instant initial est étudié dans la sect. 7. La section 8 contient certains compléments, notamment la validité uniforme pour tout $t>0$ de l'approximation sous des hypothèses plus restreintes. Finalement, dans la section 9 nous étudions un problème de perturbation des valeurs propres qui donne une nouvelle démonstration de nos résultats dans le cas particulier où l'opérateur $B$ ne dépend pas de $t$ et leś valeurs propres du problème non perturbé sont simples.

Tout le long de l'article, les raisonnements et notations se simplifient si l'on ne considère que le cas où les valeurs propres du problème non perturbé sont simples. Le lecteur pressé pourra se limiter à ce cas.

\section{2. - Une classe de problèmes linéaires d'évolution.}

Considérons un espace de Hilbert séparable $H$, identifié à son dual $H^{\prime}$ et deux espaces de Hilbert $V$ et $W$, denses dans $H$ et satisfaisant aux inclusions algébriques topologiques

$$
V \subset W \subset H \equiv H^{\prime} \subset W^{\prime} \subset V^{\prime}
$$


On se donne deux familles de formes hermitiennes continues

$$
\begin{array}{ll}
a(t ; u, v) & \text { sur } V \\
b_{1}(t ; u, v) & \text { sur } W
\end{array}
$$

telles que, pour $u$ et $v$ fixes soient des fonctions continûment différentielles de $t \in[0, T]$, $T$ fini. Elles satisfont aussi aux hypothèses de coercivité:

$$
\begin{cases}a(t ; u, u) \geqslant \gamma\|u\|_{V}^{2} ; & \gamma>0, u \in V \\ b_{1}(t ; u, u)+\lambda\|u\|_{B}^{2} \geqslant \beta\|u\|_{W}^{2} ; & \lambda, \beta>0, u \in W \\ b_{1}(t ; u, u) \geqslant 0 ; & u \in W\end{cases}
$$

Désignons par $A(t)$ et $B_{1}(t)$ les familles d'opérateurs associés aux formes $a$ et $b_{1}$ :

$$
\begin{aligned}
a(t ; u, v) & =\langle A(t) u, v\rangle ; & & A(t) \in \mathfrak{L}\left(V, \nabla^{\prime}\right) \\
b_{1}(t ; u, v) & =\left\langle B_{1}(t) u, v\right\rangle ; & & B_{1}(t) \in \mathfrak{L}\left(W, W^{\prime}\right)
\end{aligned}
$$

où les crochets expriment les produits de dualité entre $V^{\prime}$ et $V$ ou entre $W^{\prime}$ et $W$.

On considère alors l'équation différentielle opérationnelle (2.4) avee les conditions initiales $(2.5)$

$$
\begin{aligned}
& \frac{d^{2} u}{d t^{2}}+A(t) u+B_{1}(t) \frac{d u}{d t}=f(t) ; \quad f \in L^{2}\left(0, T ; W^{\prime}\right) \\
& u(0)=u^{*} \in V ; \quad \frac{d u}{d t}(0)=u^{* *} \in H .
\end{aligned}
$$

Proposition 2.1. - Le problème (2.4), (2.5) possède une solution unique appartenant aux espaces:

$$
\begin{aligned}
& u \in L^{\infty}(0, T ; \nabla) \\
& \frac{d u}{d t} \in L^{\infty}(0, T ; H) \cap L^{2}(0, T ; W)
\end{aligned}
$$

Nous ne développerons pas ici la démonstration, qui est analogue à celle de [13], chap. 3 , sect. 8 , avec les modifications évidentes. Avec des valeurs initiales appartenant à des espaces plus restreints, ce problème entre aussi dans le "cas de trois familles " de [14].

Signalons que, de façon préeise, la proposition 2.1 exprime qu'il existe une fonction $u(t)$ et une seule appartenant aux espaces (2.6) et (2.7), prenant la valeur $u^{*}$ pour $t=0$ et telle que:

$$
\int_{0}^{T}\left[a(t ; u, \psi)-\left(\frac{d u}{d t}, \frac{d \psi}{d t}\right)_{H}+b_{1}\left(t ; \frac{d u}{d t}, \psi\right)\right] d t=\int_{0}^{T}\langle f, \psi\rangle d t-\left(u^{* *}, \psi(0)\right)_{H}
$$


pour toute fonction-test $\psi$ telle que

$$
\psi \in L^{2}(0, T ; V) ; \quad \frac{d \psi}{d t} \in L^{2}(0, T ; W) ; \quad \psi(T)=0
$$

Remarque 2.1. - Au cours de la démonstration, qui se fait à l'aide des approximations de Galerkin, on voit que la solution satisfait aux estimations a priori que l'on obtient formellement en multipliant (2.4) par $d u / d t$.

Remarque 2.2. - L'opérateur $A(t)$ (resp. $B_{1}(t)$ ) peut être considéré comme un opérateur autoadjoint, non borné, de $H$ dans $H$, et $V$ (resp. $W$ ) coïncide avec le domaine de $A(t)^{\frac{1}{2}}, D\left(A^{\frac{1}{2}}\right)$ (resp. $\left.B_{1}(t)^{\frac{1}{2}}, D\left(B_{1}^{\frac{1}{2}}\right)\right)$. Cf. [15], 6.2.6.

\section{3. - Problème avec un petit paramètre.}

Dans le cadre général de la section 2 , considérons $A$ indépendant de $t$ et $f$ nul (oscillationsi libres).

Quant à l'opérateur $B_{1}$, nous supposerons qu'il a un petit paramètre en facteur et qu'il varie lentement avec $t$, plus précisément,

$$
B_{1}(t)=\varepsilon B(\tau) ; \quad \tau=\varepsilon t
$$

où $\varepsilon$ est un petit paramètre réel positif et $B(\tau)$ est défini pour tout $\tau$ positif et satisfait à

$$
\begin{aligned}
& |b(\tau ; u, v)| \leqslant M\|u\|_{W}\|v\|_{W} \\
& b(\tau ; u, u)+\lambda\|u\|_{H}^{2} \geqslant \beta\|u\|_{W}^{2}
\end{aligned}
$$

Nous supposerons aussi que $B$ dépend de $\tau$ de façon deux fois continument différentiable dans le norme de $\mathcal{L}\left(W, W^{\prime}\right)$ et que, en tant qu'opérateur non borné de $H$ dans $H, B(\tau) x$ est deux fois continument différentiable pour $x \in D(B)$.

Sans chercher les hypothèses minima, signalons que ces hypothèses ont lieu, par exemple, si $b(\tau ; u, v)$ dépend analytiquement de $\tau$ pour $u$ et $v$ fixés (cf. [15], chap. 3, par. 3).

Nous supposerons en outre que l'immersion de $V$ dans $H$ est compacte. Dans ces conditions, considerons l'equation

$$
\frac{d^{2} u}{d t^{2}}+A u+\varepsilon B(\tau) \frac{d u}{d t}=0
$$

avec les conditions initiales (2.5).

Exprimons maintenant (3.4) dans une base spéciale.

Sous les hypothèses considérées, l'opérateur $A^{-1}$ existe et est compact de $H$ dans $H$, 
is bien qu'il existe une base orthogonale des espaces $H$ et $V$ formée par les vecteurs propres $e_{i}$ de $A$, que nous supposerons normalisés dans $H$, les valeurs propres correspondantes étant:

$$
0<\omega_{1}^{2} \leqslant \omega_{2}^{2} \leqslant \ldots \leqslant \omega_{n}^{2} \leqslant \ldots \rightarrow+\infty
$$

Naturellement, plusieurs valeurs propres peuvent être égales (valeur propre multiple). Dans ce cas, nous dirons qu'un «mode" (s) est formé par l'ensemble des indices $n$ dont la valeur propre $\omega_{n}^{2}$ est la même, celle du mode $(s)$. Les indices qui correspondent aux modes seront toujours écrits entre parenthèses; par exemple (3.5) peut aussi s'écrire:

$$
0<\omega_{(1)}^{2}<\omega_{(2)}^{2}<\ldots<\omega_{(s)}^{2}<\ldots \rightarrow+\infty
$$

En multipliant (3.4), dont les différents termes appartiennent à $V^{\prime}$ par $e_{k}$ au sens de la dualité entre $V^{\prime}$ et $V$, compte tenu des propriétés d'orthogonalité des vecteurs propres, nous obtenons (3.6)-(3.8), ainsi que les conditions initiales (3.9):

$$
\begin{aligned}
& u(t)=\sum_{1}^{\infty} u_{j}(t) e_{j} \\
& \frac{d^{2} u_{k}(t)}{d t^{2}}+\omega_{k}^{2} u_{k}(t)+\varepsilon \sum_{j=1}^{\infty} b_{j k}(\tau) \frac{d u_{j}}{d t}=0
\end{aligned}
$$

où

$$
\begin{aligned}
& b_{j k}(\tau)=b\left(\tau ; e_{j}, e_{k}\right) \\
& u_{k}(0)=u_{k}^{*} ; \quad \frac{d u_{k}}{d t}(0)=u_{k}^{* *}
\end{aligned}
$$

Introduisons maintenant quelques notations qui seront utiles par la suite:

La forme $b^{d}$, ainsi que l'opérateur associé $B^{d}$ ( $d$ comme "diagonale ") sont définis formellement dans la base choisie par la matrice infinie

$$
b_{j k}^{d}(\tau)= \begin{cases}=b_{j k}(\tau) & \text { si } \omega_{j}=\omega_{k} \\ =0 & \text { sinon }\end{cases}
$$

il est clair alors que l'opérateur $B^{d}$ opère de chaque mode en lui-même.

Il est évident que l'espace $V$ peut être muni du produit scalaire exprimé par la forme $a$; il introduit une norme équivalente à la norme donnée. On peut alors introduire l'espace d'énergie $E=V \times H$ de norme

$$
\|\boldsymbol{x}\|_{E}^{2}=a(x, x)+\left\|x^{\prime}\right\|_{H}^{2} ; \quad \boldsymbol{x}=\left(x, x^{\prime}\right)
$$


Cette notation sera appropriée pour exprimer (3.4) en tant que système du premier ordre pour les deux inconnues $u, u^{\prime}$ (la virgule désignant la dérivation par rapport à $t)$.

ReMarque 3.1. - En nous limitant, pour fixer les idées, au cas $W=V$, il est clair que la perturbation $\varepsilon \rightarrow 0$ est singulière, car l'équation (3.4) pour $\varepsilon=0$ est hyperbolique, tandis que pour $\varepsilon>0$ est parabolique. Dans l'espace d'énergie, (3.4) s'écrit:

$$
\frac{d \boldsymbol{u}}{d t}+\mathscr{A}(\tau) \boldsymbol{u}=0 ; \quad \mathscr{A}=\left(\begin{array}{cc}
0 & -I \\
A & \varepsilon B(\tau)
\end{array}\right) ; \quad \boldsymbol{u}=\left(\begin{array}{c}
u \\
\frac{d u}{d t}
\end{array}\right)
$$

L'opérateur $\mathfrak{A}$ est associé à la forme

$$
\boldsymbol{a}(\tau ; \boldsymbol{u}, \boldsymbol{v})=a\left(u, v^{\prime}\right)+\varepsilon b\left(\tau ; u^{\prime}, v^{\prime}\right)-a\left(u^{\prime}, v\right)
$$

qui est coercive sur $V \times V$ pour $\varepsilon>0$ puisque

$$
\boldsymbol{a}(\tau ; \boldsymbol{u}, \boldsymbol{u})+\lambda\|\boldsymbol{u}\|_{E}^{2}=\varepsilon b\left(\tau ; u^{\prime}, u^{\prime}\right)+\lambda a(u, u)+\lambda\left\|u^{\prime}\right\|_{I}^{2}
$$

si bien que, pour $\varepsilon>0$ le problème entre dans le cadre des problèmes paraboliques (cf. par exemple [14], chap. IV).

D'autre part, pour $\varepsilon=0$, l'opérateur $A$ est anti-autoadjoint dans $E$, si bien que le problème est hyperbolique et l'existence et l'unicité relèvent du théorème de Stone; on a alors

$$
\boldsymbol{u} \in L^{\infty}(0, T ; E)
$$

si bien que le processus $\varepsilon \rightarrow 0$ fait perdre l'appartenance de $u^{\prime}$ à $L^{2}(0, T ; W$ ) (cf. (2.7)) et constitue done une perturbation singulière. Il est pourtant remarquable qu'on peut se donner la valeur initiale $\boldsymbol{u}(0) \in E$ dans les deux cas; ceci est une conséquence du fait que l'on étudie des solutions faibles; le domaine de l'opérateur $A$ est par ailleurs différent dans les cas $\varepsilon>0$ et $\varepsilon=0$.

\section{4. - Application formelle des échelles multiples.}

Cherchons la solution formelle de $(3.7),(3.9)$ sous la forme:

$$
u(t)=u^{0}(t, \tau)+\varepsilon u^{1}(t, \tau)+\ldots ; \quad \tau \in \varepsilon t
$$

uniformément valable dans un intervalle de $t$ de longueur $O\left(\varepsilon^{-1}\right)$, par exemple, $t \in[0$, $\left.L \varepsilon^{-1}\right]$. En fait, nous étudierons seulement le terme $u^{0}$ auquel nous imposerons les 
conditions initiales (3.9) soit

$$
u_{k}^{0}(0)=u_{k}^{*} ; \quad \frac{d u_{k}}{d t}(0)=u_{k}^{* *} .
$$

En introduisant l'identité

$$
\frac{d}{d t}=\frac{\partial}{\partial t}+\varepsilon \frac{\partial}{\partial \tau}
$$

dans (3.4) et en développant en puissances de $\varepsilon$ nous obtenons aux ordres $\varepsilon^{0}$ et $\varepsilon^{1}$ :

$$
\begin{aligned}
& \frac{\partial^{2} u_{0}}{\partial t^{2}}+A u_{0}=0 \\
& \frac{\partial^{2} u^{1}}{\partial t^{2}}+A u^{1}=-2 \frac{\partial^{2} u_{0}}{\partial t \partial \tau}-B \frac{\partial u_{0}}{\partial t} .
\end{aligned}
$$

En développant dans la base $e_{k}$ on peut intégrer (4.3) sous la forme (4.5), où les fonctions $\alpha^{ \pm}(\tau)$ sont pour le moment indéterminées; (4.4) devient alors (4.5 bis)

$$
\begin{aligned}
u_{k}^{0} & =\alpha_{k}^{-}(\tau) \exp \left[-i \omega_{k} t\right]+\alpha_{k}^{+}(\tau) \exp \left[i \omega_{k} t\right] \\
\frac{\partial^{2} u^{1}}{\partial t^{2}}+\omega_{k}^{2} u_{k}^{1}=2 i \omega_{k}[- & \left.\frac{d \alpha^{+}}{d \tau} \exp \left[i \omega_{k} t\right]+\frac{d \alpha^{-}}{d \tau} \exp \left[-i \omega_{k} t\right]\right]+ \\
& +\sum_{i} b_{j k} i \omega_{j}\left[-\alpha \exp \left[i \omega_{j} t\right]+i \omega_{j} \alpha^{-} \exp \left[-i \omega_{j} t\right]\right]
\end{aligned}
$$

Mais dans le second membre de (4.5 bis) les exposants exp $\left[ \pm i \omega_{k} t\right]$ ne doivent pas apparaitre; sinon, l'intégration ferait intervenir des termes en $t \exp \left[ \pm i \omega_{k} t\right]$ qui, pour $t$ de l'ordre de $\varepsilon^{-1}$ feraient que $u^{0}$ et $\varepsilon u^{1}$ seraient du même ordre, si bien que le développement (4.1) serait illusoire.

Nous obtenons alors pour la détermination des $\alpha$ les équations (4.6) où pour chaque $k$ la somme du second membre est étendue aux indices $j$ appartenant au même mode que $k$. Ceci peut s'écrire sous la forme (4.7), en notation symbolique pour toutes les composantes, mais il est clair que ce système infini se découple en des systèmes finis pour chaque mode.

$$
\begin{aligned}
& \frac{d \alpha_{k}^{ \pm}}{d \tau}=\frac{-1}{2} \tilde{\Sigma} b_{j k} \alpha_{j}^{ \pm} \\
& \frac{d \alpha^{ \pm}}{d \tau}=\frac{-1}{2} B^{a}(\tau) \alpha^{ \pm} .
\end{aligned}
$$

Les conditions initiales pour $\alpha^{ \pm}$se déduisent de (4.2) et (4.5), ce qui donne (4.9), ou, compte tenu de (4.7), en désignant par $\alpha_{\left(\frac{1}{8}\right)}^{ \pm}$la projection de $\alpha^{ \pm}$sur les indjces cor- 
respondant au mode $(s),(4.10)$

$$
\begin{aligned}
& \left\{\begin{array}{l}
u_{k}^{*}=\alpha_{k}^{+}(0)+\alpha_{\vec{k}}(0) \\
u_{k}^{* *}=i \omega\left[\alpha_{k}^{+}(0)-\alpha_{k_{i}}^{-}(0)\right]+\varepsilon\left[\frac{d \alpha_{k}^{+}}{\partial \tau}(0)+\frac{d \alpha_{k}}{d \tau}(0)\right]
\end{array}\right. \\
& \left\{\begin{array}{l}
u_{(s)}^{0}=\alpha_{(s)}^{+}(0)+\alpha_{(s)}^{-}(0) \\
u_{(s)}^{* *}=i \omega\left[\alpha_{(s)}^{+}(0)-\alpha_{(s)}^{-}(0)\right]-\frac{\varepsilon}{2} B^{d}(0)\left[\alpha_{(s)}^{+}(0)+\alpha_{(s)}^{-}(0)\right] .
\end{array}\right.
\end{aligned}
$$

Nous voyons que les conditions initiales se découplent aussi pour les différents modes.

$I l$ est clair que, pour le calcul de $u^{0}$, on pourra se limiter au cas où un seul mode, $(s)$, est excité à l'instant initial, e'est à dire où $u_{k}^{*}$ et $u_{k}^{* *}$ sont non nuls seutement pour les $k$ appartenant au mode $(s)$. Le problème de la recherche de $u^{0}, \alpha^{ \pm}$est alors de dimension finie, dans l'espace correspondant au mode (s).

Les fonctions $\alpha^{ \pm}(\tau)$ sont bien définies et régulières pour $\tau \in[0, L]$ car, pour $\varepsilon$ suffisamment petit, (4.10) est inversible.

D'autre part, dans le sous-espace de dimension finie (s), les normes de $V, W$ et $H$ sont équivalentes, si bien qu'il existe une constante $O_{(s)}$ qui dépend du mode $(s)$ excité à l'instant initial, telle que, pour tout $t \in\left[0, L \varepsilon^{-1}\right]$,

$$
\left\|u^{0}\right\|_{v}, \quad\left\|\frac{\partial u^{0}}{\partial t}\right\|_{V}, \quad\left\|\frac{d u^{0}}{d t}\right\|_{V}, \quad\left\|\frac{\partial u^{0}}{\partial \tau}\right\|_{V} \leqslant C_{(s)}\left\|_{\boldsymbol{u}^{*}}\right\|_{E} .
$$

Ici et dans la suite, $C_{(s)}$ désignera des constantes dépendant du mode $(s)$, qui peuvent varier d'une formule à une autre. Nous pouvons maintenant énoncer le théorème d'estimation de l'erreur:

THÉoRìme 4.1. - Si la donnée initiale $\boldsymbol{u}^{*}=\left(u^{*}, u^{* * *}\right)$ porte seulement sur le mode $(s)$, il existe $C_{(s)}$ telle que:

$$
\left\|\boldsymbol{u}(t)-\boldsymbol{u}_{0}(t, \tau)\right\|_{E} \leqslant O_{(8)} \varepsilon\left\|\boldsymbol{u}^{*}\right\|_{E} \quad \forall t \in\left[0, L \varepsilon^{-1}\right]
$$

Les deux sections qui suivent sont consacrées à la démonstration de ce résultat. Le eas où tous les modes sont excités à l'instant initial est abordé dans la section 7 .

\section{5. - Cas où un seul mode est éxcité à l'instant initial. Estimation de $u^{1}$.}

L'équation (4.4) pour le terme $u^{1}$ devient, compte tenu de ce qui précède:

$$
\frac{\partial^{2} u^{1}}{\partial t^{2}}+A u^{1}+\left(B-B^{d}\right) \frac{\partial u^{0}}{\partial t}=0
$$


Pour estimer la différence entre $u$ et $u^{0}$, introduisons un terme de fermeture $y$ dans la suite formelle (4.1), défini par

$$
u(t)=u^{0}(t, \tau)+\varepsilon u^{1}(t, \tau)+\varepsilon y(t)
$$

Le terme $u^{1}$, qui doit satisfaire à (5.1), n'a pas été entièrement déterminé, car sa dépendance en $\tau$ et ses valeurs initiales n'ont pas été fixées. En fait, nous déterminerons $u^{1}$ sous une forme simple et nous estimeron $u^{1}$ en nous appuyant sur sa forme; ensuite, le terme $y$ qui, d'après la comparaison de (5.2) et (4.1) est probablement "petit ", sera majoré par une estimation a priori énergétique.

Cherchons $u^{1}(t, \tau)$ de la forme (5.3) qui, en vertu de (5.1), nous donne (5.4) pour la recherche de $v \pm(\tau)$

$$
\begin{aligned}
& u^{1}(t, \tau)=v^{+}(\tau) \exp \left[i \omega_{(s)} t\right]+v^{-}(\tau) \exp \left[-i \omega_{(s)} t\right] \\
& \left(A-\omega_{(s)}^{2}\right) v^{ \pm}(\tau)= \pm i \omega_{(s)}\left(B(\tau)-B^{\alpha}(\tau)\right) \alpha^{ \pm}(\tau)
\end{aligned}
$$

Considérons (5.4) comme une équation opérationnelle dans l'espace $H$, l'inconnue étant $v^{ \pm}$et $\tau$, un paramètre. Pour résoudre cette équation, il suffit d'appliquer l'alternative de Fredholm ( $A^{-1}$ est compact), compte tenu que $\omega_{(g)}^{2}$ est une valeur propre de $A$, et que les seules composantes non nulles de $\alpha^{ \pm}$sont celles qui correspondent au mode (s). Les $e_{i}$ appartiennent au domaine de $A$ et par conséquent à $D(B(\tau))$ (cf. remarque 2.2), si bien que le second membre de (5.4) est un élément de $H$ dont les composantes correspondantes an mode $(s)$ sont nulles. Par ailleurs, cet élément dépend de façon continument différentiable de $\tau$. Puisque $A-\omega_{(s)}^{2}$ a un semi-inverse borné dans l'espace orthogonal à celui des vecteurs propres (cf. remarque 5.1 plus loin), $v^{ \pm}(\tau)$ existent et dépendent de $\tau$ de façon continument différentiable dans la norme de $H$.

Puisque $\alpha$, ainsi que $\left(B-B^{a}\right) \alpha$ sont entièrement déterminés par les valeurs initiales, en vertu de la linéarité, il s'ensuit que pour $\tau \in[0, L]$, soit $t \in\left[0, L \varepsilon^{-1}\right]$, il existe une constante $C_{(s)}$ telle que:

$$
\left\|u^{1}\right\|_{B} \leqslant O_{(s)}\left\|\boldsymbol{u}^{*}\right\|_{\underline{H}}
$$

D'autre part, d'après (5.4), $v$ appartient à $D(A)$, donc à $V$. En multipliant scalairement par $v^{ \pm}$dans $H$, il vient:

$$
a\left(v^{ \pm}, v^{ \pm}\right)=\omega_{(s)}^{2}\left\|v^{ \pm}\right\|_{H}^{2} \pm i \omega_{(s)}\left(\left(B-B^{d}\right) \alpha^{ \pm}, v^{ \pm}\right)_{H}
$$

On peut alors minorer le premier membre à l'aide de la coercivité, et majorer le second membre à l'aide de (5.5). On en déduit que:

$$
\left\|u^{\mathrm{I}}\right\|_{V} \leqslant C_{(s)}\left\|\boldsymbol{u}^{*}\right\|_{E}, \quad t \in\left[0, L \varepsilon^{-1}\right]
$$


REMARQUe 5.1. - L'utilisation de la base formée par les vecteurs propres permet de tronver une expression explicite du semi-inverse $S$ de $A-\omega_{(8)}^{2}$ qui peut être utilisée comme méthode alternative pour obtenir les estimations (5.5) et (5.6). (La précédente admettait que $D(A) O D(B))$ on a:

$$
\begin{aligned}
A \sum x_{i} e_{i} & =\sum \omega_{i}^{2} x_{i} e_{i} \\
\left(A-\omega_{(s)}^{2}\right) \sum x_{i} e_{i} & =\sum\left(\omega_{i}^{2}-\omega_{(s)}^{2}\right) x_{i} e_{i}
\end{aligned}
$$

si bien que, si l'on se donne $y$ avec les composantes $y_{(s)}$ nulles, e'est à dire orthogonal aux vecteurs propres associés à la valeur propre $\omega_{(3)}^{2}$, en multipliant ses composantes par $\left(\omega_{i}^{2}-\omega_{(s)}^{2}\right)^{-1}$ on obtient un élément $S y$ bien déterminé, appartenant au dit orthogonal et tel que $\left(A-\omega_{(s)}^{2}\right) S y=y$.

Par ailleurs, puisque $\omega_{i} \rightarrow \infty$ si $i \rightarrow \infty$, il existe une constante $C_{(s)}$ telle que:

$$
\|S y\|_{V}^{2}=\sum \omega_{i}^{2}\left(\omega_{i}^{2}-\omega_{(s)}^{2}\right)^{-2}\left|y_{i}\right|^{2} \leqslant O_{(s)} \sum\left|y_{i}\right|^{2} \omega_{i}^{-2}
$$

si bien que $S$ est borné de $V^{\prime}$ dans $V$.

Finalement, compte tenu de l'expression explicite de $u^{1}(5.3)$, écrivons l'équation différentielle satisfaite par $u^{1}$ en termes de la dérivée totale par rapport à $t$. Compte tenu de (5.7), (5.1) devient (5.8) où $f_{1}$ et $f_{2}$ sont définis par (5.9), qui satisfait à l'estimation (5.10) en vertu de ce qui précède:

$$
\begin{aligned}
& \frac{d^{2}}{d t^{2}}=\frac{\partial^{2}}{\partial t^{2}}+2 \varepsilon \frac{\partial^{2}}{\partial t \partial \tau}+\varepsilon^{2} \frac{\partial^{2}}{\partial \tau^{2}} \\
& \frac{d^{2} u^{1}}{d t^{2}}+A u^{1}=-\left(B-B^{d}\right) \frac{\partial u_{0}}{\partial t}+\varepsilon f_{1}(t)+\varepsilon^{2} f_{2}(t) \\
& f_{1}(t)=2 \frac{\partial^{2} u^{1}}{\partial t \partial \tau} ; \quad f_{2}(t)=\frac{\partial^{2} u^{1}}{\partial \tau^{2}} \\
& \left\|f_{i}(t)\right\|_{H} \leqslant O_{(s)}\left\|\boldsymbol{u}^{*}\right\|_{B}, \quad i=1,2 ; t \in\left[0, L \varepsilon^{-1}\right] .
\end{aligned}
$$

On peut écrire de même l'équation satisfaite par $u^{0}$ (4.3), compte tenu de (5.7) s'écrit (5.11)

De (4.5) et (4.7) on déduit (5.12), si bien que (5.11) s'écrit (5.13) où $f_{3}$ est une fonction régulière de $t$ à valeurs dans $H$ et satisfaisant à (5.14).

$$
\begin{aligned}
& \frac{d^{2} u^{0}}{d t^{2}}+A u_{0}=2 \varepsilon \frac{\partial^{2} u^{0}}{d t \partial \tau}+\varepsilon^{2} \frac{\partial^{2} u^{0}}{\partial \tau^{2}} \\
& -2 \frac{\partial^{2} u^{0}}{\partial \tau^{2}}=B^{a} \frac{\partial u^{0}}{\partial t} \\
& \frac{d^{2} u^{0}}{\partial t^{2}}+A u_{0}=-\varepsilon B^{d} \frac{\partial u^{0}}{\partial t}+\varepsilon^{z} f_{3}(t) \\
& \left\|f_{3}(t)\right\|_{\pi} \leqslant C_{(s)}\left\|\boldsymbol{u}^{*}\right\|_{E} ; \quad t \in\left[0, L \varepsilon^{-1}\right] .
\end{aligned}
$$




\section{6. - Estimation du terme complémentaire $y$.}

A la différence du terme $u^{1}$ dont les estimations (5.5) et (5.6) ont été obtenues en utilisant la forme explicite de $u^{1}$, pour $y$ nous obtiendrons une estimation a priori basée sur l'équation et les conditions initiales satisfaites par $y$. En remplaçant le second membre de (5.2) dans (3.4) et en tenant compte de (5.8) et (5.13) on voit que $y$ satisfait à l'équation (6.1) et, par ailleurs, aux conditions initiales (6.2)

$$
\begin{gathered}
\frac{d^{2} y}{d^{2} t^{2}}+A y+\varepsilon B \frac{d y}{d t}=B\left(\frac{\partial u^{0}}{\partial t}-\frac{d u^{0}}{d t}\right)-\varepsilon B \frac{d u^{1}}{d t}-\varepsilon\left(f_{1}+f_{3}\right)-\varepsilon^{2} f_{2} \\
y(0)=\boldsymbol{u}^{1}(0)
\end{gathered}
$$

Transformons le premier terme du second membre de (6.1), qui s'écrit $-\varepsilon \partial u_{0} / \partial \tau$. Il est, d'après l'expression explicite de $u^{0}$, une fonction régulière de $t$ à valeurs dans le sous-espace de dimension finie correspondant au mode (s). Cette fonction est done à valeurs dans $V$, donc dans $W$, et en faisant opérer $B$ on voit que le premier terme $d u$ second membre de (6.1) est une fonction régulière à valeurs dans $W^{\prime}$. II en va de même du second terme, en vertu de (5.6). En conclusion, l'équation satisfaite par $y$ peut s'écrire sous la forme (6.3) où $f_{2}$ et $f_{4}$ sont des fonetions régulières à valeurs dans $W^{\prime}$, qui satisfont par ailleurs à l'estimation (6.4). Par ailleurs de (5.6) et (6.2) on voit que les valeurs initiales satisfont à (6.5)

$$
\begin{aligned}
& \frac{d^{2} y}{d t^{2}}+A y+\varepsilon B(\varepsilon t) \frac{d y}{d t}=\varepsilon f_{4}(t)+\varepsilon^{2} f_{2}(t) \\
& \left\|f_{i}\right\|_{W^{\prime}} \leqslant C_{(s)}\left\|\boldsymbol{u}^{*}\right\|_{E} ; \quad i=2,4 ; t \in\left[0, L \varepsilon^{-1}\right] \\
& \|\boldsymbol{y}(0)\|_{E} \leqslant C_{(s)}\left\|\boldsymbol{u}^{*}\right\|_{E}
\end{aligned}
$$

Nous voyons que $y$ satisfait à une équation analogue à celle de départ, (3.4), mains non homogène, le second membre ayant $\varepsilon$ en facteur. Il est donc normal que, pendant un temps $O\left(\varepsilon^{-1}\right), y$ soit de l'ordre de l'unité; e'est ce que nous allons prouver.

Multiplions (6.3) par $d y / d t$ (ef. remarque 2.1), il vient:

$$
\frac{1}{2} \frac{d}{d t}\left(\left\|\frac{d y}{d t}\right\|_{\|}^{2}+a(y, y)\right)+\varepsilon b\left(\varepsilon t ; \frac{d y}{d t}, \frac{d y}{d t}\right)=\varepsilon\left\langle f, \frac{d y}{d t}\right\rangle
$$

où

$$
f=f_{4}+\varepsilon f_{1}
$$

En intégrant de 0 à $t$ nous obtenons (6.7); ensuite, en minorant le premier membre à l'aide de la propriété de coereivité (3.3) et en majorant le second membre, nous 
obtenons (6.8) et (6.9).

$$
\begin{gathered}
\|\boldsymbol{y}(t)\|_{E}^{2}-\|\boldsymbol{y}(0)\|_{E}^{2}+2 \varepsilon \int_{0}^{t} b\left(\varepsilon \sigma, y^{\prime}, y^{\prime}\right) d \sigma \leqslant 2 \varepsilon \int_{0}^{t}\|f\|_{W^{\prime}}\left\|\frac{d y}{d t}\right\|_{F} d \sigma \\
\|\boldsymbol{y}(t)\|_{E}^{2}-\|\boldsymbol{y}(0)\|_{E}^{2}+2 \varepsilon \beta \int_{0}^{t}\left\|\frac{d y}{d t}\right\|_{W}^{2} d \sigma-2 \varepsilon \lambda \int_{0}^{t}\left\|\frac{d y}{d t}\right\|_{z}^{2} d \sigma \leqslant \varepsilon \int_{0}^{t}\left(\frac{1}{\beta}\|f\|_{W^{\prime}}^{2}+\beta\left\|\frac{d y}{d t}\right\|_{W}^{2}\right) d \sigma \\
\|\boldsymbol{y}(t)\|_{E}^{2} \leqslant\|\boldsymbol{y}(0)\|_{E}^{2}+2 \varepsilon \lambda \int_{0}^{t}\|\boldsymbol{y}\|_{E}^{2} d \sigma+\frac{\varepsilon}{\beta} \int_{0}^{t}\|f\|_{W^{\prime}}^{2} d \sigma
\end{gathered}
$$

Mais, en vertu de (6.4) le terme contenant $f$ de la dernière inégalité rest borné pour $t \in\left[0, L \varepsilon^{-1}\right]$; en utilisant aussi $(6.5)$, nous avons $(6.10)$ et, en appliquant l'inégalité de Gronwall, (6.11), d'où (6.12).

$$
\begin{aligned}
& \|\boldsymbol{y}(t)\|_{E}^{2} \leqslant 2 \varepsilon \lambda \int_{0}^{t}\|\boldsymbol{y}(\sigma)\|_{I}^{2} d \sigma+O_{(s)}\left\|\boldsymbol{u}^{*}\right\|_{E}^{2} \\
& \|\boldsymbol{y}(t)\|_{E}^{2} \leqslant O_{(s)}\left\|\boldsymbol{u}^{*}\right\|_{E}^{2} \exp [2 \varepsilon \lambda t] \\
& \|\boldsymbol{y}(t)\|_{E} \leqslant O_{(s)}^{\prime}\left\|\boldsymbol{u}^{*}\right\|_{E} ; \quad t \in\left[0, L \varepsilon^{-1}\right]
\end{aligned}
$$

Les estimations (5.5), (5.6) et (6.12) prouvent le théorème 4.1.

\section{7. - Cas où tous les modes sont excités à l'instant initial.}

Puisque le problème est linéaire, le principe de superposition permet d'étendre les résultats qui précèdent au cas où un nombre fini de modes sont excités à l'instant initial. Le théorème 4.1 reste alors valable en mettant comme constante $C$ au second membre de sup. des constantes correspondantes aux modes excités.

Supposons à présent que tous les modes sont excités, et que les valeurs initiales $u^{*}$ et $u^{* *}$ sont des éléments quelconques de $V$ et $H$ (cf. (2.5)), soit, dans la norme d'énergie

$$
\boldsymbol{u}(0)=\boldsymbol{u}^{*} \in E
$$

Nous allons construire une expression approchée $u^{0}$ qui approche uniformément de $u$ pour $t \in\left[0, L \varepsilon^{-1}\right]$. A cet effet, étant donné $\delta$ arbitrairement, on décompose $\boldsymbol{u}^{*}$ sous la forme:

$$
\left\{\begin{array}{l}
\boldsymbol{u}^{*}=\boldsymbol{u}_{\delta}^{*}+\left(\boldsymbol{u}^{*}-\boldsymbol{u}_{\delta}^{*}\right) \\
\left\|\boldsymbol{u}^{*}-\boldsymbol{u}_{\delta}^{*}\right\|_{E}<\delta / 2
\end{array}\right.
$$


telle que $\boldsymbol{u}_{\delta}^{*}$ porte seulement sur un nombre fini de modes. Construisons alors la solution $u_{\delta}^{0}$ associée aux valeurs initiales tronquées $\boldsymbol{u}_{\delta}^{*}$, qui porte évidemment sur un nombre fini de modes. Dans ces conditions, nous arons:

THस́OR:̀me 7.1. - Etant donné $\delta$, pour $\varepsilon$ suffisamment petit, on a:

$$
\left\|\boldsymbol{u}(t)-\boldsymbol{u}_{\delta}^{0}(t)\right\|_{E} \leqslant \delta ; \quad t \in\left[0, L \varepsilon^{-1}\right]
$$

La démonstration est immédiate. Si l'on désigne par $u_{\delta}$ la solution exacte correspondante à la valeur unitiale $u_{\delta}^{*}$, en vertu du théorème 4.1, valable pour un nombre fini de modes, avec une constante de $C_{\delta}$, on aura:

$$
\left\|\boldsymbol{u}_{\delta}-\boldsymbol{u}_{\delta}^{0}\right\|_{E} \leqslant \frac{\delta}{2} ; \quad t \in\left[0, L \varepsilon^{-1}\right]
$$

si

$$
\varepsilon<\frac{\delta}{2 O_{\delta}\left\|\boldsymbol{u}^{*}\right\|_{E}}
$$

D'autre part, $\left\|\boldsymbol{u}-\boldsymbol{u}_{\delta}\right\|_{E}$ est une fonction non croissante de $t$, car $w=u-u_{\delta}$ est la solution exacte correspondante aux valeurs initiales $\boldsymbol{u}^{*}-\boldsymbol{u}_{\delta}^{*}$, si bien que l'on a l'estimation (analogue à (6.7); on a utilisé la seconde formule (3.3)):

$$
\|\boldsymbol{w}\|_{E}^{2} \leqslant\|\boldsymbol{w}(0)\|_{E}^{2}+2 \varepsilon \int_{0}^{t} b\left(w^{\prime}, w^{\prime}\right) d \sigma \leqslant\left\|\boldsymbol{u}^{*}-\boldsymbol{u}_{\delta}^{*}\right\|_{E}^{2}
$$

ce qui achève la démonstration du théorème.

Il est clair que la solution approchée $u_{\delta}^{0}$ correspond à des valeurs initiales portant sur un nombre fini de modes, nombre qui doit être choisi en fonction de $\delta$, mais qui est indépendant de $\varepsilon$. En fait, le nombre fini de modes peut être choisi aussi grand que l'on veut (tout en étant fini) et la borne supérieure des valeurs de $\varepsilon$ pour que le théorème 7.1 soit valable, dépendra du nombre de composantes choisi. Dans la pratique, en prenant un nombre suffisamment grand (fini) de modes et $\varepsilon$ suffisamment petit, on est sûr d'aroir une approximation valable de la solution exacte.

On peut se demander si l'approximation sera améliorée en prenant un $u^{0}$ qui satisfasse exactement anx conditions initiales (7.1), c'est à dire en prenant en considération tous les modes. Cette question nous semble ouverte, mais certaines considérations permettent de penser que la réponse serait négative. Considérons par exemple le cas où $W=V, \lambda$ dans (3.3) est nul et $B$ ne dépend pas de $\tau$. De (4.7) et (5.11) on déduit que l'équation satisfaite par $u^{0} \mathrm{~s}^{\prime} e ́ c r i t:$

$$
\frac{d^{2} u^{0}}{d t^{2}}+\left[A+\frac{\varepsilon^{2}}{4}\left(B^{d}\right)^{2}\right] u_{0}+\varepsilon B^{d} \frac{d u^{0}}{d t}=0
$$


et il est facile de voir que le terme en $\varepsilon^{2}$ introduit une perturbation singulière. Il suffit de constater que le domaine de $B^{a}$ est le même que celui de $A$ ou $B$, puisqu'alors le domaine de l'opérateur entre crochets de (7.3) serait plus petit que celui de $A$. La perturbation singulière peut par ailleurs empêcher d'imposer les conditions initiales (7.1) à $u^{0}$.

Etudions donc la forme $b^{d}$. Pour $u \in V$, comme les projections sur les modes $u_{(s)}$ sont orthogonales dans $V$, on a:

$$
b^{d}(u, u)=\sum_{\{s\}} b\left(u_{(s)}, u_{(s)}\right) \geqslant \sum_{(s)} \beta\left\|u_{\{s)}\right\|_{V}^{2}=\beta\|u\|_{r}^{2}
$$

si bien que $b^{a}$ est coercive sur $V$. De même, en utilisant le principe de polarisation, on déduit que $b^{d}$ est sesquilinéaire bornée sur $V$. En conséquence $D\left(B^{a}\right)=D(A)$ c.q.f.d.

REMARque 7.1. - Il n'y a pas de contradiction entre les considérations qui précèdent et celles de [9] sect. 2.4. Ces dernières concernent la convergence des séries intervenant dans les expressions de chacun des coéfficientss $u_{E}(t)$.

\section{8. - Compléments et applications.}

Le caractère dissipatif de l'opérateur $B$ dans l'équation (3.4) suggère que la solution approchée $u^{0}$ pourrait être uniformément valable dans tout le demi-axe $t$ positif. On voit en effet, dans la théorie du Centrage, que si l'équation centrée a la solution $u=0$ asymptotiquement stable, l'approximation est uniformément valable (of. par exemple [16], [17]). Dans le problème qui nous ici, nous ignorons si les conclusions des théorèmes 4.1 et 7.1 restent valables pour tout $t \geqslant 0$ sous les hypothèses considérées.

Considérons le cas où $W=V, B$ ne dépend pas de $\tau$ et (3.3) a lieu avec $\lambda=0$. Les équations (4.6) pour chaque mode sont des équations linéaires de coéfficients constants dans un espace de dimension finie, en plus, la solution nulle est exponentiellement stable (cf. (7.4)), si bien qu'il existe une constante $K$, qui dépend en général du mode $(s)$ telle que:

$$
\|\alpha(\tau)\| \leqslant\|\alpha(0)\| C_{(s)} \exp [-k \tau], \quad \tau \geqslant 0
$$

On en déduit facilement que les estimations (4.11), (5.5), (5.6), (5.10), (5.14) et (6.4) ont lieu pour tout $t \geqslant 0$ en ajoutant le facteur $\exp (-K \varepsilon t)$ au second membre. L'inégalité (6.9) donne alors, pour tout $t \geqslant 0$ :

$$
\|y(t)\|_{E}^{2} \leqslant\|y(0)\|_{E}^{2}+\varepsilon \beta \int_{0}^{t} C_{(s)}\left\|\boldsymbol{u}^{*}\right\|_{E}^{2} \exp [-K \varepsilon t] \leqslant C_{(s)\rangle}^{\prime}\left\|\boldsymbol{u}^{*}\right\|_{E}^{2}
$$


E. SANCHez-Palencla: Justification de la méthode des échelles multiples, etc. 173

En combinant ce résultat avec l'estimation de $u^{1}$ on a prouvé le:

THस́onìne 8.1. - Dans le cas où $B$ ne dépend pas de $\tau$, (3.3) a lieu avec $\lambda=0$ et $W=V$, les estimations des théorèmes 4.1 et 7.1 sont valables pour tout $t \geqslant 0$.

Sans développer les calculs, signalons que les résultats des sections qui précèdent s'appliquent aussi à des équations de la forme:

$$
u^{\prime \prime}+A u+\varepsilon B(\tau) u^{\prime}+\varepsilon O(\tau) u=0
$$

où $O(\tau)$ est un opérateur associé à la forme $e(\tau ; u, v)$ sesquilinéaire sur $W$, (non nécessairement coercive).

Nous n'avons pas réussi à établir pour des équations du type (3.4) des résultats sur le comportement des trajectoires dans l'espace des phases analogues à ceux de [18] pour des équations différentielles dans des espaces de Banach. Le problème de l'étude de la forme des trajectoires au voisinage de l'origine (noeud, foyer ...), qui a un intérêt en acoustique (cf. [9], sect. 2.6) semble done ouvert.

Nos résultats justifient, au moins pour la première approximation $u^{0}$, les calculs d'échelles multiples pour les problèmes de vibration de corps élastiques avec un petit terme de viscoélasticité instantanée (c'est à dire, à mémoire instantanée) de [9]. Ce problème correspond en fait au cas particulier $W=V$ du présent travail. Plus généralement, si l'on considère un corps élastique $\Omega$ hétérogène, qui soit viscoélastique seulement dans un sous-ensemble $\Omega_{1}$ de $\Omega$, on est conduit à une équation du type (3.4) avec $W$ différent de $V$. Avee les notations de [9] (signalons pour mémoire que les $e_{i j}$ ont les composantes du tenseur de taux de déformation et que $u^{i}$ on $v^{i}$ sont les composantes de deux vecteurs déplacement possibles pour le solide; les $a_{i j k h}^{m}$ sont les coéfficients d'élasticité pour $m=1$ et de viscoélasticité pour $m=2$ ) on peut définir:

$$
\begin{aligned}
& (u, v)_{H}=\int_{\Omega} u_{i} v_{i} d x \\
& (u, v)_{V}=a(u, v)=\int_{\Omega} a_{i j k h}^{1} e_{i j}(u) e_{k h}(v) d x \\
& b(u, v)=\int_{\Omega_{1}} a_{i j k h}^{2} e_{i j}(u) e_{k h h}(v) d x \\
& (u, v)_{W}=b(u, v)+(u, v)_{H}
\end{aligned}
$$

et l'on est dans le cadre que nous étudions ici.

\section{9. - Lien avec la méthode de pérturbation des valeurs propres.}

Considérons le cas où l'opérateur $B$ ne depend pas de $\tau$ et les valeurs propres $\omega_{j}^{2}$ sont simples. On pent se proposer de chercher des solutions exponentielles (complexes) en $t$, ce qui conduit à la recherche de valeurs propres et vecteurs propres 
perturbés par $\varepsilon$. On peut dans le cas où les valeurs propres du problème non perturbé sont simples, retrouver les résultats précédents, ce qui donne une nouvelle justification de la méthode des échelles multiples pour ce type de problèmes. Les raisonnements qui suivent sont exactement analogues à ceux que nous avions utilisés dans [19], sect. 3 et 4 , à propos d'un problème de résonance acoustique. Celà nous dispensera de donner ici certains détails.

Si l'on cherche solution de l'équation (3.4) de la forme $v \exp [-i \omega t]$ (un calcul analogue peut être fait pour $\exp [i \omega t])$. Il vient:

$$
-\omega^{2} v+(A-i \varepsilon B) v=0
$$

Si $\omega_{j}^{2}$ est une valeur propre simple de l'opérateur non perturbé $A$, l'inverse $d u$ perturbé $(A-i \varepsilon \omega B)^{-1}$ est un opérateur compact de $H$ dans $H$ qui dépend de façon holomorphe de $\varepsilon$ et de $\omega$ pour $|\varepsilon|$ et $\left|\omega-\omega_{j}\right|$ suffisamment petits (le fait que $\omega_{i}^{2}$ soit une valeur propre de $A$ ne joue aucun rôle pour le moment; on aurait pu prendre $\omega_{j}$ réel positif quelconque). (9.1) devient

$$
(A-i \varepsilon \omega B)^{-1} v=\frac{1}{\omega^{2}} v
$$

Il s'agit évidemment de trouver $\omega(\varepsilon)$ prenant la valeur $\omega_{j}$ pour $\varepsilon=0$, telle que (9.2) ait une solution $v(\varepsilon)$ non nulle. Nous voyons que $\omega(\varepsilon)^{-2}$ est une valeur propre de l'opérateur du premier membre de (9.2), qui dépend lui-même de $\omega(\varepsilon)$. De la théorie de perturbations holomorphes (cf. [15], chap. 7 ou [20], chap. 8) on déduit que, puisque $\omega_{j}^{-1}$ est une valeur propre simple de l'opérateur de (9.2) pour $\varepsilon=0$, la valeur propre $\lambda(\varepsilon, \omega)$ existe, est simple et dépend de façon holomorphe de $\varepsilon$ et $\omega$ pour $|\varepsilon|$ et $\left|\omega-\omega_{i}\right|$ suffisamment petits. La recherche de $\omega(\varepsilon)$ se réduit à la résolution de l'équation implicite

$$
F(\varepsilon, \omega) \equiv \lambda(\varepsilon, \omega)-\frac{1}{\omega^{2}}=0
$$

qui est satisfaite par $\varepsilon=0, \omega=\omega_{j}$. En dérivant dans ce point, comme $\lambda$ ne dépend pas de $\omega$ pour $\varepsilon=0$, (évident d'après la forme de l'opérateur de (9.2)) on a:

$$
\frac{\partial F}{\partial \omega}\left(0, \omega_{j}\right)=\frac{2}{\omega_{j}^{3}} \neq 0
$$

si bien que, d'après le théorème des fonctions implicites, $\omega(\varepsilon)$ existe bien, est holomorphe pour $|\varepsilon|$ suffisamment petit, et prend la valeur $\omega_{j}$ pour $\varepsilon=0$. Nous désignerons cette fonction par $\omega_{j}(\varepsilon)$. On voit aussi que les vecteurs propres correspondants, $e_{j}(\varepsilon)$ sont des fonctions holomorphes bien définies pour $|\varepsilon|$ suffisamment petit (on normalise par exemple par $\left\|e_{j}(\varepsilon)\right\|_{B}=1$ ). Ceci nous conduit à l'existence de solutions de (3.4) de la forme

$$
u(j, \varepsilon, t)=e_{j}(\varepsilon) \exp \left[-i \omega_{j}(\varepsilon) t\right]
$$


qui peuvent être considérées comme les solutions équivalentes dans le problème perturbé aux solutions:

$$
e_{j} \exp \left[-i \omega_{j} t\right]
$$

du problème non perturbé.

Les fonctions $e_{j}(\varepsilon)$ et $\omega_{j}$ étant holomorphes, admettent les développements (9.6) et (9.7); prenons comme expression approchée de (9.4) celle qu'on obtient en ne retenant que les termes d'ordre zéro en $\varepsilon$ dans (9.6) et d'ordre un en $\varepsilon$ dans (9.7), e'est à dire, (9.8):

$$
\begin{aligned}
& e_{j}(\varepsilon)=e_{j}+\varepsilon e_{j}^{*}+\ldots \\
& \omega_{j}(\varepsilon)=\omega_{j}+\varepsilon \omega_{j}^{*}+\ldots \\
& u_{\mathrm{appr}}(j, \varepsilon, t)=e_{j} \exp \left[-i \omega_{j} t\right] \exp \left[-i \omega_{j}^{*} \varepsilon t\right]
\end{aligned}
$$

Il s'agit maintenant de comparer la différence entre $u$ et $u_{\text {appr }}$. En écrivant (9.9), un calcul immédiat basé sur le fait que $\omega_{j}$ est réel, montre que l'on a (9.10).

$$
\begin{aligned}
& u-u_{\mathrm{appr}}=\left(u-e_{j} \exp \left[-i \omega_{j}(\varepsilon) t\right]\right)+\left(e_{j} \exp \left[-i \omega_{j}(\varepsilon) t\right]-u_{\mathrm{appr}}\right) \\
& \left\|\boldsymbol{u}-\boldsymbol{u}_{\mathrm{appr}}\right\|_{E} \leqslant C_{j} \varepsilon ; \quad t \in\left[0, L \varepsilon^{-1}\right]
\end{aligned}
$$

Remarquons que $u$ et $u_{\text {apps }}$ ne satisfont pas nécessairement aux mêmes conditions initiales, mais (9.10) étant valable pour $t=0$ et les solutions exactes étant non croissantes dans la norme d'énergie, on voit que la différence entre $u_{\text {apdr }}$ et la solution exacte qui satisfait aux mêmes conditions initiales est $O(\varepsilon)$ pour tout $t<L \varepsilon^{-1}$.

En conséquence nous retrouverons le théorème 4.1 si l'on prouve que les solutions $(9.8)$ et $u^{0}$ de la section 4 coïncident (ou, à la rigueur, si leur différence est $O(\varepsilon)$ pour $\left.t \leqslant L_{\varepsilon^{-1}}\right)$. Pour constater qu'il en est ainsi, il suffit de faire un calcul formel analogue à celui de [20], sect. 37 .

Introduisons les développements $(9.6),(9.7)$ dans $(9.1)$, ce qui donne une identité en $\varepsilon$; en identifiant à zéro le coéfficient de $\varepsilon$, nous obtenons (9.11) et en multipliant scalairement dans $H$ par $e_{j}$, compte tenu de ce que $A$ est autoadjoint et $\omega_{j}^{2}$ sa valeur propre correspondante à $e_{j}$, on a (9.12) qui, compte tenu de (4.5) et (4.6), permet d'identifier la solution approchée de (9.8) avec le terme en exp $\left[-i \omega_{j} t\right]$ de (4.5), c.q.f.d.

$$
\begin{aligned}
& -2 \omega_{j} \omega_{j}^{*} e_{j}-\omega_{j}^{2} e_{j}^{*}-i \omega_{j} B e_{j}+A e_{j}^{*}=0 \\
& -2 \omega_{j}^{*}=i b\left(e_{j}, e_{j}\right) \Rightarrow \omega_{j}^{*}=(-i / 2) b_{j j}
\end{aligned}
$$

REMARQUE 9.1. - Si $\omega_{j}^{2}$ est une valeur propre multiple de l'opérateur non perturbé $A$, la méthode précédente ne s'applique plus car la valeur propre peut bifurquer pour 
$\varepsilon>0$ et la fonction $F$ de (9.3) n'est pas en général, holomorphe. Le raisonnement par l'absurde de [19], théorème 3.1 s'applique pourtant, ce qui permet de prouver que, pour $\varepsilon$ suffisamment petit, le problème pertubé possède au moins une fréquence propre $\omega_{j}(\varepsilon)$ qui converge vers $\omega_{j}$ lorsque $\varepsilon$ tend vers zéro. Mais dans ce cas on ne peut en déduire une justification des calculs d'échelles multiples.

\section{BIBLIOGRAPHIE}

[1] N. Bogoltovbov - I. Mrtropolskx, Les méthodes asymptatiques en théorie des oscillations non linéaires, Gauthier-Villars, Paris (1962).

[2] J. D. CoLE, Perturbation methods in applied mathematies, Blaisdell, New York (1968).

[3] M. Roseav, Equations différentielles, Masson, Paris (1976).

[4] J. A. Monrison, Comparaison of the modified method of averaging and the two-variable expansion procedure, S.I.A.M. Review, 8 (1966), pp. 66-85.

[5] I. MitropoLSKI - B. I. Molseenkov, Legons sur la méthode de centrage pour des équations aux dérivées partielles (en russe), Kiev (1969).

[6] P. P. ZABREIKo - YU. I. FETISov, The small parameter method for hyperbolic equations, Differ. Equat. (1975), pp. 626-634; Trad. de Differ. Uravn., 8 (1972), pp. 823-834.

[7] M. Roseau, Méthodes asymptotiques et oscillations non linéaires de systèmes physiques dont l'espace de configuration est de dimension infinie (à parâttre, volume en hommage à M. T. Vogel).

[8] P. L. CHоw, Asymptotic solutions of inhomogeneous initial boundary value problems for weakly non linear partial differential équations, S.I.A.M. Journ. Appl. Math., 22 (1972), pp. 629-647.

[9] S. Cernead - E. Sanchez-Palencia, Sur les oscillations libres des corps élastiques légèrement viscoélastiques, Jour. Mécan., 15 (1976), pp. 237-263.

[10] G. N. BOJADZIEV - R. W. LARDNER, Asymptotie solutions of partial differential equations with damping and delay, Quart. Appl. Math., 33 (1975), pp. 205-214.

[11] E. L. REISs, On multivariable asymptotic expansions, S. I. A. M. Review, 13 (1971), pp. $189-196$.

[12] J. L. Lions, Perturbations singulières dans les problèmes aux limites et en controle optimal, Springer, Berlin (1973).

[13] J. L. LIons - E. MAGENEs, Problèmes aux limites non homogènes et applications, vol. I, Dunod, Paris (1968).

[14] J. L. Lrons, Equations différentielles opérationnelles et problèmes aux limites, Springer, Berlin (1961).

[15] T. Kaтo, Perturbation theory for linear operators, Springer, Berlin (1966).

[16] V. M. VoLosov, Averaging on an unbounded interval, Soviet Math. Dokl., 3 (1962), pp. 113-114; Trad. de Dokl. Akad. Nauk, 145 (1962), pp. 965-966.

[17] C. BANFI, Sull'approssimazione di processi non stazionari in meccanica non lineare, Boll. Un. Mat. Ital., 22 (1967), pp. 442-450.

[18] E. Sanchez-PaLencta, Methode de Centrage. Estimation de l'erreur et comportement des trajectoires dans l'espace des phases, Int. Jour. Non Linear Mech., 11 (1976), pp. 251-263.

[19] E. SanChEZ-PALENCIA, Perturbations spectrales liées à la vibration d'un corps élastique dans l'air, à paraître dans les Actes des Journées Mathématiques sur les perturbations singulières, Lyon, Décembre 1976, Lecture Notes in Mathematics 594.

[20] K. O. FRIEDRICHS, Spectral Theory of Operators in Hilbert Space, Springer, New York (1973). 